\title{
Real-Time Neural Signals of Perceptual Priming with Unfamiliar Geometric Shapes
}

\author{
Joel L. Voss ${ }^{1}$ and Ken A. Paller ${ }^{2}$ \\ ${ }^{1}$ Beckman Institute for Advanced Science and Technology, University of Illinois Urbana-Champaign, Urbana, Illinois 61801, and ${ }^{2}$ Interdepartmental \\ Neuroscience Program and Department of Psychology, Northwestern University, Evanston, Illinois 60208
}

Perceptual priming is a type of item-specific implicit memory that is distinct from explicit memory. Neural signals of the processing responsible for perceptual priming can be difficult to isolate due to concurrent conceptual processing and explicit recognition. We successfully identified neural correlates of perceptual priming by using minimally meaningful, difficult-to-recognize, kaleidoscope images. Human participants were required to quickly indicate the number of colors present in each stimulus, and priming was shown by faster and more accurate visual discriminations for repeated compared with initial presentations. Electroencephalographic responses linked with this differential perceptual fluency were identified as negative potentials $100-300$ ms poststimulus onset. Furthermore, different potentials recorded during initial presentations were indicative of perceptual learning, in that their amplitude predicted the magnitude of later priming. These electrophysiological findings show that the degree of perceptual learning engaged upon first encountering a novel visual stimulus predicts the degree of perceptual fluency experienced when the stimulus is processed a second time. It is thus possible to isolate multiple neural processing stages relevant to perceptual priming by using real-time measures of relevant neurophysiological activity in conjunction with experimental circumstances that limit the contaminating influences of other neurocognitive events.

\section{Introduction}

Priming refers to facilitated behavioral responses to a specific stimulus, often due to facilitated perceptual processing following repetition (Richardson-Klavehn and Bjork, 1988; Roediger, 1990). Priming can occur without conscious awareness of memory retrieval and is therefore considered an expression of "implicit memory," which is functionally and neurally distinct from expressions of "explicit memory" such as recall (Gabrieli, 1998; Schacter and Buckner, 1998; Henson, 2003; Squire, 2004; Schacter et al., 2007).

Priming in the visual modality has been associated with changes in neural processing in extrastriate visual cortex (Wiggs and Martin, 1998; Henson, 2003; Schacter et al., 2007). These neural effects vary in a posterior-to-anterior gradient along the ventral visual processing stream (Ungerleider and Haxby, 1994), with more posterior effects reflecting priming for physical stimulus features (perceptual priming) and more anterior effects reflecting facilitated processing of semantic/conceptual features, or conceptual priming (Schacter et al., 2007).

Uncertainty remains regarding how perceptual priming occurs (Grill-Spector et al., 2006). One complicating issue is that preexisting knowledge can influence perceptual priming (Peterson

\footnotetext{
Received Jan. 24, 2010; revised April 29, 2010; accepted May 24, 2010.

Financial support was provided by a Beckman Institute Postdoctoral Fellowship award to J.L.V. and by a grant from the National Science Foundation (BCS-0818912). Many thanks to Heather Lucas and Susan Florczak for help with data collection.

Correspondence should be addressed to Joel L. Voss, Beckman Institute, University of Illinois Urbana-Champaign, 405 North Mathews Avenue, Urbana, IL 61801. E-mail: joelvoss@illinois.edu.

DOI:10.1523/JNEUROSCI.0403-10.2010

Copyright $\odot 2010$ the authors $\quad 0270-6474 / 10 / 309181-08 \$ 15.00 / 0$
}

and Enns, 2005; Boehm et al., 2006). Indeed, even at early stages in the ventral stream (extrastriate cortex) neurons can show category-selective activity reflecting influences from preexisting memory representations (Allison et al., 1999). Another difficulty is that neural activity accompanying explicit recognition may occur in tandem with, and be difficult to separate from, neural activity responsible for implicit memory (Slotnick and Schacter, 2006; Voss and Paller, 2008a). One way to address these limitations is to rely on stimuli without preexisting memory representations, such that priming can occur largely independent of conceptual processing (Musen and Treisman, 1990; Schacter et al., 1990; DeSchepper and Treisman, 1996).

Priming for familiar stimuli occurs with diminished neural activity in ventral visual cortex and left inferior prefrontal cortex (Schacter and Buckner, 1998; Henson, 2003; Schacter et al., 2007), similar to "repetition suppression" effects found in recordings from single neurons (Desimone, 1996; Brown and Aggleton, 2001). In contrast, priming for unfamiliar stimuli occurs with greater neural activity in many of these same brain regions (Henson et al., 2000; Fiebach et al., 2005; Soldan et al., 2008), and these effects have been attributed to perceptual learning. However, it is unclear whether activity enhancements for unfamiliar stimuli reflect perceptual learning, implicit memory, or explicit memory.

We tested priming for novel geometric kaleidoscope images in a way that complemented our previous studies of event-related potentials (ERPs) produced in response to these stimuli (Voss and Paller, 2009a,b). Our prior results provided impetus for using divided-attention study conditions to limit explicit memory and for using neural measures to categorize explicit versus 
implicit memory. The present design thus allowed us to characterize electrophysiological correlates of perceptual priming while minimizing concurrent conceptual processing and explicit memory. We also assessed perceptual learning during initial kaleidoscope presentation by identifying ERPs that predicted later priming, as in prior studies that found differences between activity predictive of priming versus explicit memory for words (Paller et al., 1987b; Paller, 1990; Paller and Kutas, 1992; Schott et al., 2002, 2006).

\section{Materials and Methods}

Behavioral and ERP data were acquired from 16 Northwestern University students (ages 19-27 years, 12 female). All subjects were right-handed and native speakers of English. Data from three additional subjects were excluded due to excessive eye-blink artifacts (though patterns of priming in excluded subjects resembled those in other subjects).

Visual stimuli were 132 kaleidoscope images constructed using methods described in detail previously (Voss et al., 2008; Voss and Paller, 2009a). Each kaleidoscope image was constructed by deforming three, four, or five overlaid hexagons, with each hexagon having a unique, randomly selected color other than black (Fig. 1; 40 of each type). Kaleidoscopes were displayed on a black background such that they subtended $\sim 5^{\circ}$ of visual angle on a computer monitor.

A different set of kaleidoscopes was presented during each of six study test blocks. Twelve kaleidoscopes were presented consecutively during each study phase, each for $2000 \mathrm{~ms}$ at a rate of one every $2500-3500 \mathrm{~ms}$. A randomly selected spoken numeric digit was played through a speaker with onset corresponding to the onset of each kaleidoscope. In the corresponding test phase, 10 of these kaleidoscopes were shown together with 10 new kaleidoscopes, each for $1500 \mathrm{~ms}$ at a rate of one every 3000 $4000 \mathrm{~ms}$. The test did not include the first and last kaleidoscope from the study phase to guard against the possibility that memory for these two stimuli would reflect unique types of processing (primacy and recency effects). Kaleidoscopes were divided into two sets and the sets used as old images and as new images were counterbalanced across subjects. Kaleidoscopes were randomly assigned to experimental blocks for each subject.

In the study phase, subjects were required to perform a colordiscrimination task for kaleidoscopes while simultaneously performing a 1-back task for digits. Subjects thus made two behavioral responses. First, the number of colors present in the kaleidoscope image was registered using a three-choice button response $(3,4$, or 5$)$. Then, verbal report was used to register whether the number spoken on the prior trial was odd or even. Button-press response times were substantially longer than the duration of the epoch used for ERP analysis, such that study-phase ERPs were not contaminated by the verbal response (see below).

The test phase followed the study phase after a $30 \mathrm{~s}$ break during which subjects counted backwards aloud by threes from a specified number. During the test phase, subjects performed the color-discrimination task. Speed and accuracy were heavily emphasized. To encourage rapid responding, subjects were instructed to respond before an alerting tone that was played through a speaker 1000-1200 ms after stimulus onset (response deadline randomized across trials). No digits were presented in the test phase.

Continuous EEG recordings were made from 59 scalp locations using tin electrodes embedded in an elastic cap. Impedance was $<5 \mathrm{k} \Omega$. Re- cordings were digitally sampled at $1000 \mathrm{~Hz}$ with a bandpass of $0.05-200$ $\mathrm{Hz}$. Recordings were collected with a right mastoid reference, and were rereferenced offline to averaged mastoids. Stimulus-locked ERPs were calculated for each condition of interest in $1100 \mathrm{~ms}$ epochs beginning 100 ms before stimulus onset. Baseline correction was performed by subtracting the average prestimulus amplitude from each sample. An additional four channels were used for monitoring horizontal and vertical eye movements. Trials contaminated by electro-ocular or other artifacts were excluded from analysis. ERP waveforms were smoothed with a 40 $\mathrm{Hz}$ zero-phase-shift Butterworth filter for presentation purposes only.

Statistical analyses of ERP waveforms focused on amplitude values averaged over latency intervals and electrode clusters. Differences between conditions were assessed using repeated-measures ANOVA with Geisser-Greenhouse correction when necessary.

\section{Results}

\section{Behavioral findings}

We predicted that facilitated perceptual processing during the priming test would lead to faster color-discrimination responses for old (repeat) versus new kaleidoscopes. On average, responses were $\sim 22 \mathrm{~ms}$ faster for old versus new kaleidoscopes [mean $=$ 864 and $886 \mathrm{~ms}$, respectively, $t_{(15)}=2.8, p=0.01$ ]. Responses were much slower during study, when speed was not emphasized and two tasks were performed together on each trial (mean = $1294 \mathrm{~ms}, \mathrm{SE}=80$ ).

Responses to old items during the test were also more accurate than responses to new items $[$ mean $=56.1 \%$ and $47.8 \%$, respectively; $t_{(15)}=3.8, p=0.002$ ]. Accuracy during study was $46.3 \%$ $(\mathrm{SE}=1.1 \%)$, which was less accurate than during test $\left[t_{(15)}=7.2\right.$, $p<0.001]$. During both study and test, accuracy was higher than the guessing rate of 33\% ( $p$-values $<0.001)$.

Priming was thus evident as faster and more accurate colordiscrimination responses for old compared with new items. 
Study response Test response
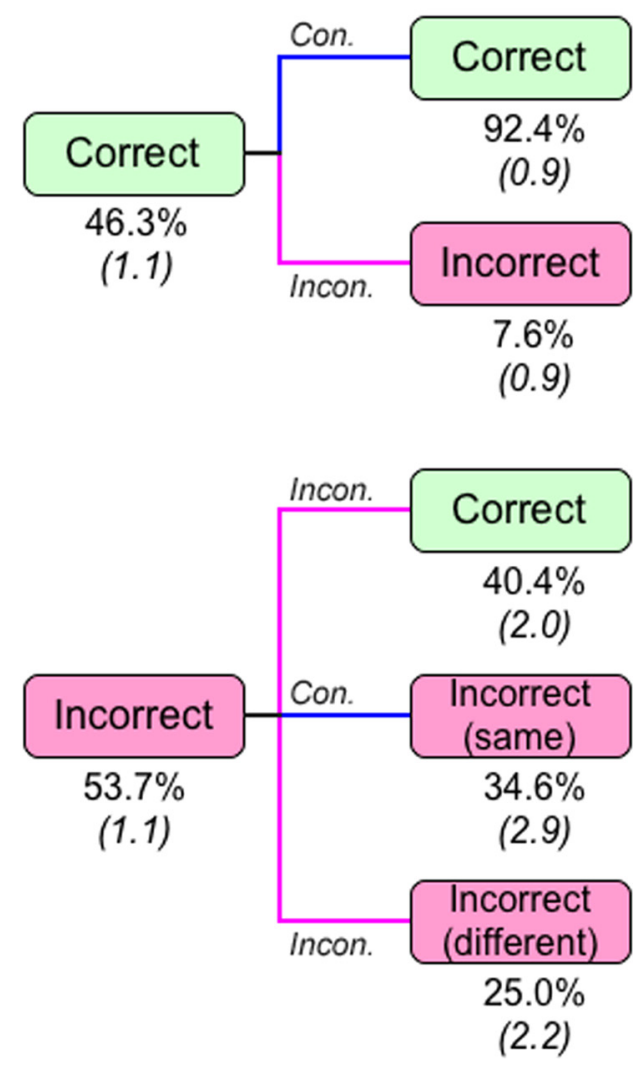

Figure 2. Color discrimination accuracy during the priming test conditional on accuracy during the study session. Blue lines indicate that the same response was made at study and test [consistent (Con.)] and magenta lines indicate that different responses were made at study and test [inconsistent (Incon.)]. For items that were incorrect at both study and test, it was possible either that the same incorrect choice was made (same) or that a different incorrect choice was made (different). SE is shown in parentheses.

However, facilitated responding during the test could conceivably be attributed to specific stimulus-response associations learned during the study phase rather than to perceptual priming per se (Horner and Henson, 2008). We assessed this possibility by examining response times as a function of study-test response consistency (Fig. 2). A different response was given at study than at test for $35.5 \%$ of the stimuli $(\mathrm{SE}=1.1 \%)$, and the response times during test for these inconsistent items averaged $863 \mathrm{~ms}$ $(\mathrm{SE}=31)$. For the remaining $64.5 \%$ of the stimuli with consistent study-test responses, response times averaged $866 \mathrm{~ms}(\mathrm{SE}=33)$. The finding that response times to old items during the test did not differ reliably based on study-test response consistency $\left[t_{(15)}=\right.$ $0.3, p=0.77]$ suggests that the response facilitation for repeated stimuli was not significantly influenced by stimulus-response association learning.

\section{Electrophysiological findings during test}

During the priming test, old kaleidoscopes produced more negative-going ERPs than did new kaleidoscopes from $\sim 100-$ $300 \mathrm{~ms}$ (Fig. 3A). This negative old/new ERP effect was most pronounced for central-posterior scalp locations, with maximum values at occipital locations (Fig. 3C). We tested the reliability of the effect from 100 to $300 \mathrm{~ms}$ for three midline electrode clusters: anterior, middle, and posterior (Fig. 3A). A main effect of condition indicated that the old/new ERP difference was reliable $\left[F_{(1,15)}=4.9, p=0.04\right]$, and a significant cluster (anterior/mid- dle/posterior $) \times$ condition $($ old $/$ new $)$ interaction indicated that the magnitude of the difference varied significantly with location $\left[F_{(1.1,16.5)}=5.1, p=0.03\right]$. The magnitude of the negative effect showed an anterior/posterior gradient, as it was least for the anterior cluster $(-0.97 \mu \mathrm{V}, p=0.13)$, intermediate for the middle cluster $(-1.1 \mu \mathrm{V}, p=0.03)$, and greatest for the posterior cluster $(-1.3 \mu \mathrm{V}, p=0.02)$.

Visual inspection of ERP waveforms revealed only minimal old/new differences after $\sim 300 \mathrm{~ms}$, and we confirmed this impression by formal assessment of ERP amplitudes for successive $200 \mathrm{~ms}$ intervals from 300 to $900 \mathrm{~ms}$, using values averaged across each of the three electrode clusters. Main effects of condition or condition $\times$ cluster interactions were not reliable ( $p$-values $>0.28)$.

Relative to new kaleidoscopes, old kaleidoscopes were associated with facilitated perceptual discriminations and negative occipital ERPs. We reasoned that if these ERP responses were closely related to learning-related priming of behavioral responses, then old kaleidoscopes associated with the fastest responses should also evoke the most negative ERPs. We therefore analyzed ERPs as a function of color-discrimination speed. Old and new kaleidoscope images were segregated into two categories, fastest and slowest, based on a median split of colordiscrimination response times performed separately for each subject. Median split was performed separately for old and new response times so that the number of old and new items in the fastest and slowest categories would be matched.

There were two ERP comparisons of primary interest: old versus new for the fastest responses and old versus new for the slowest responses. It is important to note that comparing ERPs in this way was advantageous in that it limited effects of gross differences in response times on observed ERP differences. In other words, if we were to compare old fastest to old slowest, for instance, we would expect to see ERPs related to priming mixed in with ERPs related to the differential speed of motor planning and execution. In the current analyses, average differences in response times between old and new items were only $\sim 2 \%$ of the average response time for old and new items for both the fastest and slowest categories. Therefore, old/new comparisons made separately for the fastest and slowest categories could yield ERP correlates of priming with relative insensitivity to ERP correlates of motor factors.

For the fastest category, the average response time at test was $726 \mathrm{~ms}(\mathrm{SE}=26)$ for old items and $744 \mathrm{~ms}(\mathrm{SE}=24)$ for new items. For the slowest category, the average response time at test was $1002 \mathrm{~ms}(\mathrm{SE}=36)$ for old items and $1028 \mathrm{~ms}(\mathrm{SE}=31)$ for new items. Response times were reliably faster for old compared with new items [main effect of repetition $F_{(1,15)}=4.6, p=0.05$ ], and the magnitude of the old/new difference did not vary reliably for the fastest versus slowest categories [repetition $\times$ condition interaction $F_{(1,15)}=0.64, p=0.43$ ], suggesting similar priming magnitudes.

Negative old/new ERP effects for the fastest and slowest categories (Fig. 4) were similar to overall effects (Fig. 3), but effects were more pronounced for the fastest category compared with the slowest category. For the fastest category, negative old/new effects lasted from $\sim 100$ to $400 \mathrm{~ms}$ (Fig. 4A) and were evident at widespread recording locations with an occipital focus (Fig. 4D). In contrast, negative old/new effects for the slowest category were small in amplitude and restricted in time (Fig. $4 B$ ) and space (Fig. $4 F$ ).

To quantitatively compare these old/new ERP effects, we examined difference amplitudes for the fastest and slowest catego- 
ries for the 100-300 ms interval and for the anterior, middle, and posterior electrode clusters. A main effect of condition indicated that the negative ERP old/new effect was significantly larger for the fastest compared with the slowest category $\left[F_{(1,15)}=6.3, p=0.02\right.$; nonsignificant condition $\times$ cluster interaction $F_{(2,30)}=$ 1.24]. Differences between the fastest and slowest categories in ERP old/new difference amplitudes were not reliable after $300 \mathrm{~ms}$ when tested in successive $200 \mathrm{~ms}$ intervals from 300 to $900 \mathrm{~ms}$ ( $p$-values $>0.17$ for all main effects of condition and condition-by-cluster interactions).

\section{Electrophysiological findings during study}

Neural activity during learning that predicts subsequent memory is known as Dm, for neurophysiological differences produced as a function of later memory performance (Paller et al., 1987a; Paller and Wagner, 2002). To assess activity during encoding that predicted later priming, we used a Dm approach to examine ERPs during the study phase as a function of response speed during the subsequent test. The two study conditions were laterfastest and later-slowest, with each individual trial categorized based on each subject's median-split on response times during the test, as above (a method similar to that used by Kounios et al., 2001). Response times for these categories did not differ significantly during study [later-fastest mean $=1260$, later-slowest mean $\left.=1268, t_{(15)}=0.6, p=0.56\right]$.

ERPs for the later-fastest category were more positive than ERPs for the later-slowest category during two latency intervals, as shown in Figure 5, $A$ and $B$. The first interval spanned $\sim 200-$ $500 \mathrm{~ms}$ and the second $\sim 600-1000 \mathrm{~ms}$ (i.e., until the end of the recording epoch). We refer to this positive ERP difference during encoding that predicted magnitude of subsequent priming as the "priming Dm." The topography of the priming Dm appeared to be right-lateralized during the earlier interval and bilateral during the later interval (Fig. 5C). It is important to note that this increased neural activity was not due to stimulus repetition. That is, the Dm approach allowed us to sort trials based on the amount of priming exhibited for the same stimulus when presented again in the priming test. This provided ERP correlates of trial-by-trial variations in perceptual learning. The priming Dm thus provides an index of visual learning that occurred for individual stimuli rather than as a consequence of repeatedly viewing stimuli.

We chose two latency intervals of equal duration to assess the reliability of the priming Dm (200-500 ms and 600-900 ms). The primary analysis compared mean ERP amplitudes for the later-fastest and later-slowest categories averaged for the midline anterior, middle, and posterior electrode clusters separately for the two latency intervals. For the 200-500 ms interval, neither the main effect of condition nor the condition-by-cluster interaction were reliable ( $p$-values $>0.30$ ). For the 600-900 ms interval, a main effect of condition $\left[F_{(1,15)}=5.45, p=0.03\right]$ and nonsignificant condition-by-cluster interaction $\left[F_{(2,30)}=0.11, p=0.90\right]$ indicated that later-fastest ERPs were significantly more positive than later-slowest ERPs for the three electrode clusters.

Because of the apparent lateralization of ERP effects from 200 to $500 \mathrm{~ms}$, an additional analysis tested mean amplitudes for two lateral electrode clusters (Fig. 5). For the 200-500 ms interval, there was a marginal condition-by-cluster interaction $\left[F_{(1,15)}=\right.$ $4.3, p=0.06$ ] due to reliably greater amplitudes for later-fastest than later slowest for the right cluster $\left[t_{(15)}=2.3, p=0.04\right]$ but not the left cluster $\left[t_{(15)}=0.74, p=0.47\right]$. For the $600-900 \mathrm{~ms}$ interval, the condition-by-cluster interaction was nonsignificant $\left[F_{(1,15)}=1.57, p=0.23\right]$.

\section{No effects of stimulus color/geometric complexity}

We performed a supplementary analysis that separately assessed responses to kaleidoscopes comprised of three, four, or five colors (Fig. 1) to determine whether these subsets of visual stimuli, which varied in color/geometric complexity, could have differed in their contributions to the aforementioned behavioral effects. Response times did not differ significantly for three-, four-, and five-color items during study (mean $=1247,1270$, and $1256 \mathrm{ms,}$ respectively; all pairwise $p$-values $>0.40$ ), for old items during the test (mean $=858,859$, and $871 \mathrm{~ms}$, respectively; all pairwise $p$-values $>0.39$ ), and for new items during the test (mean $=871$, 891 , and $894 \mathrm{~ms}$, respectively; all pairwise $p$-values $>0.12$ ). Likewise, accuracy did not differ for three-, four-, and five-color items during study (mean $=45.9 \%, 48.7 \%$, and $44.6 \%$, respectively; all pairwise $p$-values $>0.39$ ), for old items during the test (mean $=$ $57.8 \%, 55.0 \%$, and $55.6 \%$, respectively; all pairwise $p$-values $>0.61$ ), and for new items during the test (mean $=49.3 \%$, $49.6 \%$, and $44.1 \%$, respectively; all pairwise $p$-values $>0.24$ ). These findings thus indicate that no subset of kaleidoscope im- 


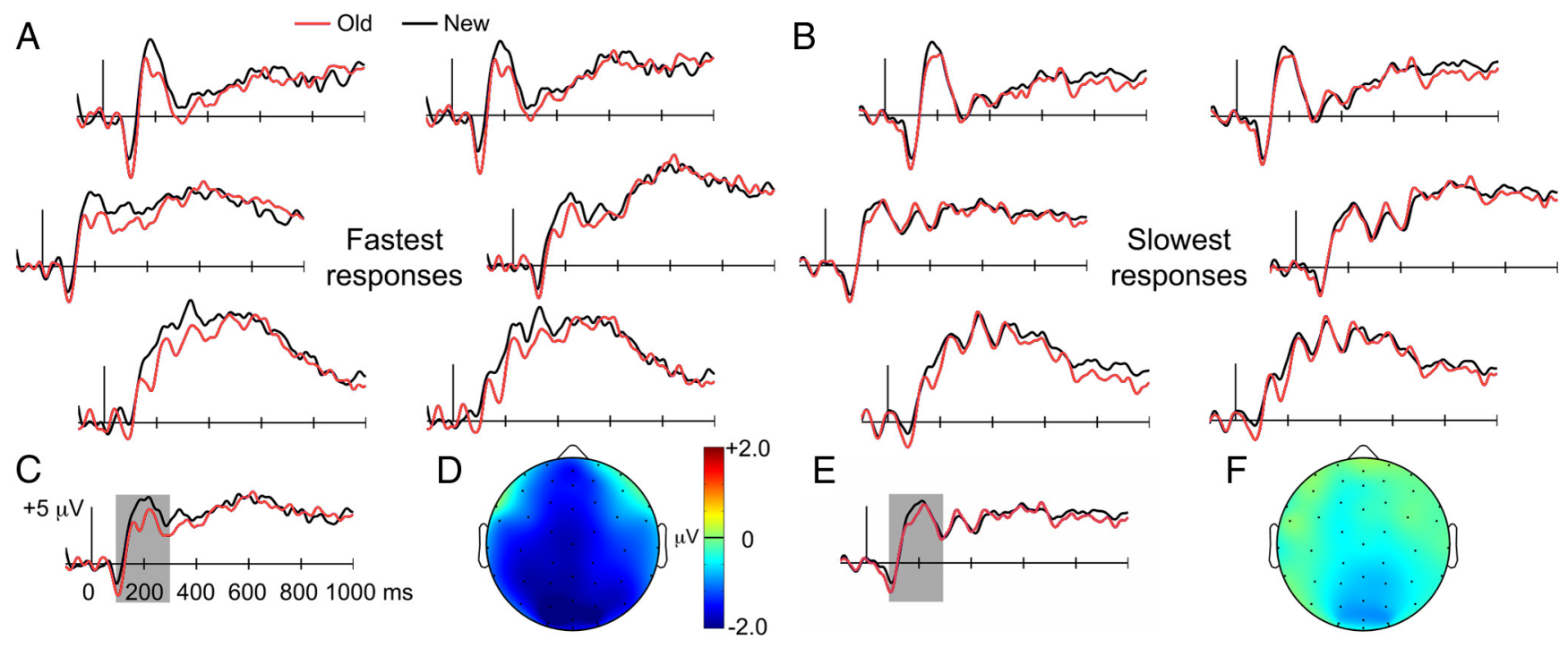

Figure 4. Negative old/new ERP effects were maximal for the fastest responses during the priming test. $A, B$, ERPs are presented as in Figure 3 for old and new items given the fastest responses during the priming test $(\boldsymbol{A})$ and given the slowest responses during the priming test $(\boldsymbol{B})$, based on median-split. $\boldsymbol{C}-\boldsymbol{F}$, ERPs averaged across all recording sites and old/new topography for the $100-300 \mathrm{~ms}$ interval are shown for the fastest responses $(\boldsymbol{C}, \boldsymbol{D})$ and the slowest responses $(\boldsymbol{E}, \boldsymbol{F})$.

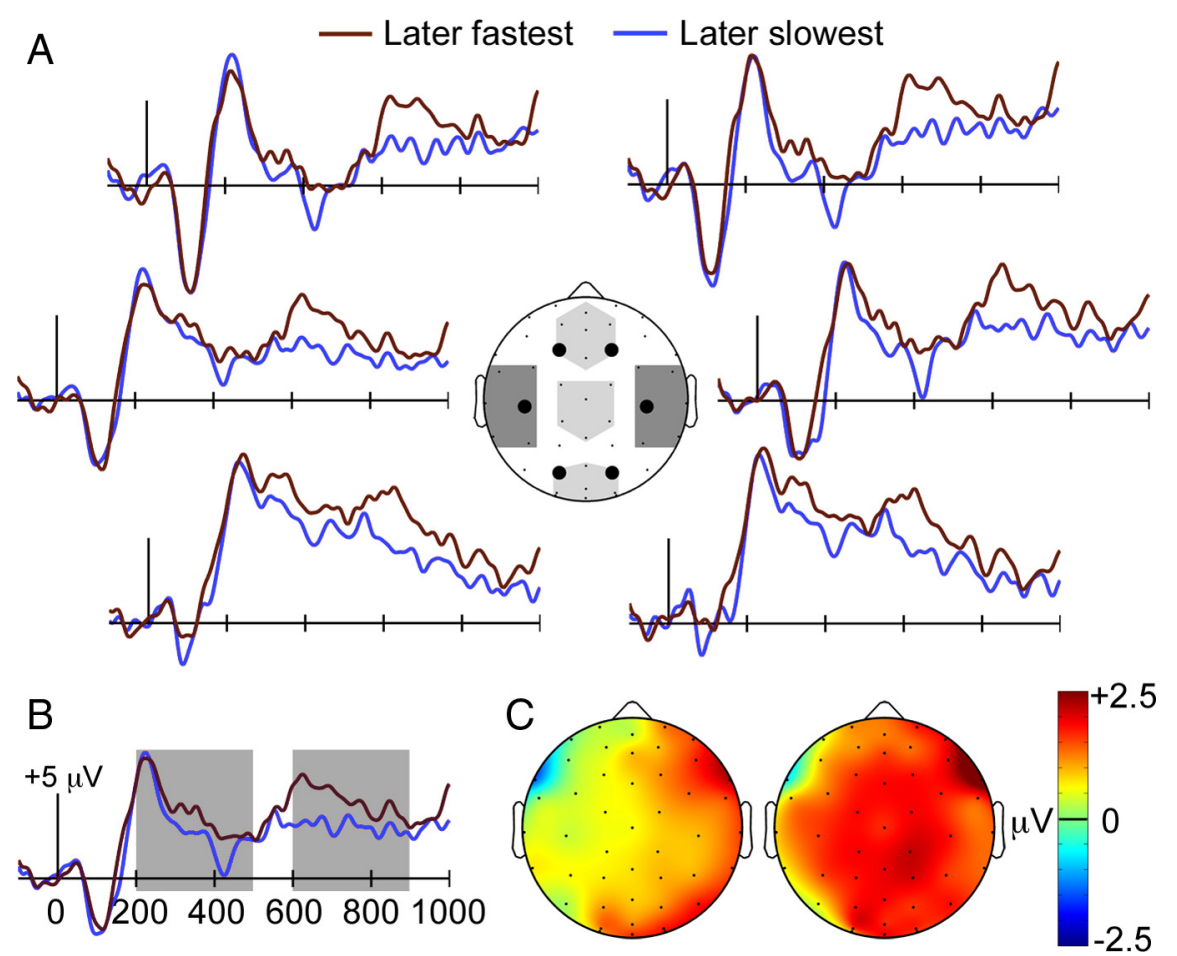

Figure 5. Priming Dm effects. $A$, ERPs during study are shown for items that subsequently elicited the fastest responses during the priming test and for items that subsequently elicited the slowest responses. Light shading on the head diagram indicates the three midline clusters used for statistical analyses, and dark shading indicates the two lateral clusters (left and right). $\boldsymbol{B}$, ERPs are shown averaged for all recording sites, and shading indicates the $200-500 \mathrm{~ms}$ and $600-800 \mathrm{~ms}$ latency intervals used for statistical assessment. $C$, Topography of ERP difference for later-fastest versus later-slowest conditions is plotted for the same two latency intervals, $200-500 \mathrm{~ms}$ at left and $600-800 \mathrm{~ms}$ at right.

ages based on number of colors carried the effects of priming on response times and on accuracy.

We performed the same subdivision of items based on color/ geometric complexity for members of the fastest and slowest categories that were assessed during the test phase. Old and new items in the fastest category included approximately equal numbers of kaleidoscopes comprised of three colors $36.9 \%$ and
$36.3 \%$, respectively; $p=0.76$ ), four colors $(32.4 \%$ and $31.7 \%$, respectively; $p=0.81)$ and five colors $(30.7 \%$ and $31.9 \%$, respectively; $p=0.51)$. Likewise, old and new items in the slowest category included approximately equal numbers of kaleidoscopes comprised of three colors $(29.6 \%$ and $29.8 \%$, respectively; $p=0.92$ ), four colors $(34.5 \%$ and $35.3 \%$, respectively; $p=0.73)$, and five colors $(35.9 \%$ and $34.9 \%$, respectively; $p=0.59$ ), indicating that old/new comparisons for fastest and slowest categories were not contaminated by differences in the color complexity of kaleidoscope images.

Finally, we performed the analogous assessment of items in the later-fastest and later-slowest categories during the study phase that were used to identify the priming Dm. Later-fastest and later-slowest categories were approximately matched in terms of their inclusion of three-color $(36.9 \%$ and $29.6 \%$, respectively; $p=$ $0.12)$, four-color $(32.4 \%$ and $34.5 \%$, respectively; $p=0.55$ ) and five-color (30.7\% and $35.9 \%$, respectively; $p=0.16$ ) kaleidoscope images, indicating that ERP comparisons between later-fastest and laterslowest items were not confounded by differences in stimulus color complexity. We thus conclude that color/geometric complexity did not contribute to the aforementioned behavioral or ERP effects.

\section{Discussion}

Novel kaleidoscope images were presented during an implicit memory test that required decisions based on the color content of each stimulus. Priming was evident as faster and more accurate decisions for repeat compared with novel stimuli. Electrophysiological correlates of priming appeared as negative deflections 
from $\sim 100-300 \mathrm{~ms}$, when ERPs were less positive for repeat than for novel stimuli, especially at occipital scalp locations. We also found an association between fluency and the magnitude of these negative old/new ERP effects, in that trials with the fastest colordiscrimination responses were associated with the greatest old/ new ERP differences.

Using the Dm approach, positive ERPs were identified as electrophysiological correlates of visual learning. The priming Dm, right lateralized from $\sim 200-500 \mathrm{~ms}$ and bilateral from $\sim 600-$ $900 \mathrm{~ms}$, is consistent with a priming Dm found with fMRI, which included activity increases in parietal cortex especially on the right (Schott et al., 2006). Collectively, our results suggest that greater perceptual learning on the first encounter with a novel item (positive priming Dm) produces a memory representation that enables more fluent processing of the same item in a subsequent encounter (negative old/new effect).

Do negative old/new effects in the current experiment really reflect implicit as opposed to explicit memory? Because processing responsible for both types of memory can occur in either type of memory test (Richardson-Klavehn and Bjork, 1988; Voss and Paller, 2008a), special strategies and converging sources of evidence are needed to address this question. Negative old/new effects similar to those reported here were associated with perceptual priming in two prior reports. In one, perceptual priming for novel faces was examined under conditions in which the concurrent retrieval of explicit memories for those faces was negligible, and negative old/new effects were identified using two different types of priming measure (Paller et al., 2003). In the other, we described negative old/new effects in conjunction with implicit perceptual fluency for repeated kaleidoscopes in a recognition test (Voss and Paller, 2009a). On the basis of several findings, we inferred that subjects taking a two-alternative forcedchoice recognition test could accurately discriminate old kaleidoscopes without being aware of the accuracy of their decisions - they guessed correctly without experiencing either familiarity or recollection, a phenomenon we termed "implicit recognition" (Voss et al., 2008; Voss and Paller, 2009a). When kaleidoscopes supported strong explicit recognition instead, old/new effects were quite distinct from those found here (Voss and Paller, 2009b). Indeed, expressions of explicit memory have generally been associated with positive old/new effects sometimes referred to as late positive complex or LPC potentials (Friedman and Johnson, 2000; Rugg and Curran, 2007; Voss and Paller, 2008b).

Figure 6 provides a juxtaposition of ERP correlates of three memory phenomena that can be triggered by kaleidoscope repetition: perceptual priming (from the current experiment), recognition based on perceptual fluency without awareness (implicit recognition), and kaleidoscope recognition based on explicit memory in the form of familiarity. Old/new effects in the current experiment bear strong similarities to those associated with implicit recognition (Voss and Paller, 2009a), particularly in topography and polarity, with some shift in timing, despite the differences in experimental design, including type of memory test. Yet, these negative old/new effects putatively associated with priming are strikingly distinct from the positive old/new effects associated with explicit memory. Positive old/new effects were virtually absent in the ERP contrasts for priming and for implicit recognition, in keeping with the notion that memory performance in the current experiment was implicit, occurring without awareness of stimulus repetition or explicit memory retrieval. Although explicit memory was not tested here, accuracy was very low in a yes-no recognition test with divided-attention study conditions similar to those used here (Voss et al., 2008), and inten-
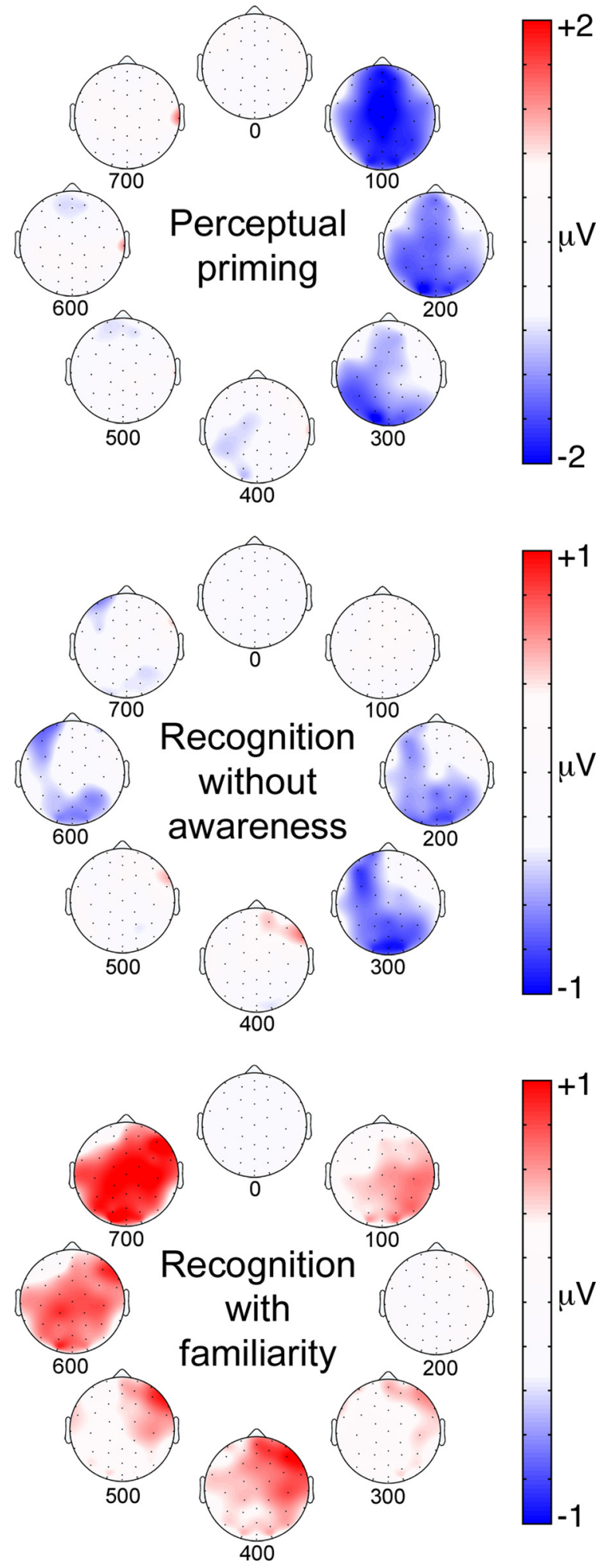

Figure 6. ERP correlates of perceptual priming, recognition without awareness (implicit recognition), and familiarity-based recognition for kaleidoscopes. Topographic plots are shown for each of the three memory types averaged for successive $100 \mathrm{~ms}$ intervals starting at stimulus onset. ERP differencevalues are indicated by coloration. Perceptual priming plots show old/newERP differences for the fastest responses, as in Figure 5. Recognition plots are modified with permission from Voss and Paller (2009a), their Figure 3. 
tional encoding with overt retrieval demands were only used in the prior design. Collectively, these considerations justify the interpretation that explicit retrieval was minimal in the current circumstances and that negative old/new effects were reflections of the processing responsible for implicit memory in the priming test.

The current findings diverge from fMRI findings showing greater neural activity for repetition of unfamiliar faces, meaningless symbols, and pseudowords, whereas repetition of familiar stimuli produces less activity (Henson et al., 2000; Fiebach et al., 2005; Soldan et al., 2008). James and Gauthier (2006) argued that the priming literature, showing reduced activity sometimes and enhanced activity other times, can best be explained by an accumulation model that describes the temporal dynamics of neural activity. These temporal dynamics are not easily resolved with fMRI methods due to their poor temporal resolution, but may be amenable to study with ERPs. In our study, repetition of novel stimuli was associated with negative old/new effects, which might indicate diminished neural activity with repetition. Critically, priming appeared to be associated with changes in ERP amplitude, not ERP latency, consistent with activity suppression models and inconsistent with the accumulation model.

Why have repeated stimuli produced such inconsistent patterns of neural activity in the literature? We propose that the divergence across studies reflects neural differences between implicit and explicit memory. That is, activity enhancements associated with repetition of novel stimuli in some previous experiments could have reflected explicit memory, if the novelty of unfamiliar stimuli prompted explicit retrieval (even though task instructions did not emphasize explicit remembering). Observing negative repetition effects (both negative ERP old/new effects and fMRI deactivations) may require negligible explicit memory. Explicit retrieval was not strongly discouraged in previous studies that found positive repetition effects with unfamiliar stimuli (Henson et al., 2000; Fiebach et al., 2005; Soldan et al., 2008). Indeed, the first neuroimaging study to identify increased ventral visual activity for priming of unfamiliar objects also showed highly accurate explicit recognition in the same circumstances, with concomitant hippocampal activation for both priming and recognition attributed to explicit retrieval (Schacter et al., 1995). Consistent with our interpretation, two experiments using unfamiliar stimuli similar to those used here identified repetition enhancements in ventral visual cortex at delays of $\sim 30 \mathrm{~s}$ when subjects made accurate recognition judgments to demonstrate explicit retrieval (Buffalo et al., 2006; Bellgowan et al., 2009). In contrast, negative repetition effects were identified for these objects when they appeared incidentally during an implicit memory test (van Turennout et al., 2000).

Memory processing engaged during repetition can clearly vary based on factors such as stimulus familiarity and behavioral demands. Therefore, the nature of the memory processing (explicit vs implicit) may play the most important role in determining the sign of observed repetition effects (positive vs negative) rather than any one of these factors individually.

It is important to note that the negative ERP correlates of perceptual priming reported here must be interpreted tentatively with respect to whether they indicate repetition-induced reductions in brain activity. The issue is that the sign of ERP effects (positive or negative) is not direct evidence for more or less brain activity, and converging evidence must instead be used to inform the interpretation of directionality. The negative ERP effects attributed to priming were occipital in focus and onset rapidly, thus appearing as negative modulations of early, object-sensitive ERP components related to activity in object-sensitive visual cortex measured with fMRI (Schendan and Lucia, 2010). Furthermore, occipital-negative ERP effects similar to those reported here have been identified in conditions that also produce reductions in object-sensitive visual cortex activity measured with fMRI (Schott et al., 2002, 2006). The available evidence thus fits well with the interpretation of priming-related negative ERP effects reported here as signals of reduced cortical activity, but direct evidence for this proposal should be provided by other means in future studies.

The current findings have implications for prevailing models of priming. Schacter et al. (2007) proposed a posterior-toanterior gradient in ventral visual cortex corresponding to a gradient from object-specific (perceptual) to amodal (conceptual) priming. Reduced cortical activity in ventral cortex is hypothesized to reflect repetition-induced tuning of neural responses to salient/stable stimulus features (Wiggs and Martin, 1998). Prefrontal cortex contributions are necessary to relate ventral cortex effects to current behavioral goals and action plans (Schacter et al., 2007). Priming is thus a reflection of representation tuning coupled with control-process facilitation (Horner and Henson, 2008). These priming mechanisms may apply even for objects seen for the first time, as in our experiment, so as to support one-trial adaptation of subsequent behavioral responding, presumably without the hippocampal participation typically necessary for one-trial explicit learning (Rolls and Kesner, 2006). An intriguing possibility that warrants future attention is that task demands can bias the engagement of brain structures capable of using priming signals for different behavioral ends. For example, negative effects in ventral cortex might occur with repetition in all circumstances, whereas only some might promote the engagement of inferior prefrontal cortex for translating these signals into an action plan versus engagement of other prefrontal regions or the hippocampal system for promoting the awareness of memory in the service of overt memory judgments (potentially by modulating recurrent feedback and activity in ventral cortex; e.g., Lamme and Roelfsema, 2000).

Studying visual learning and priming using conditions that minimize explicit memory and conceptual processing, as in the present experiment, is advantageous in that this strategy permits the straightforward observation and interpretation of relevant neural processes. Using this approach, we found that a single exposure to a novel stimulus can enhance the fluency with which it is visually processed upon subsequent exposure, and we characterized neural correlates of these memory processes. This information is essential for unraveling how learning can drive expressions of memory that occur with awareness in some circumstances and without awareness in others.

\section{References}

Allison T, Puce A, Spencer DD, McCarthy G (1999) Electrophysiological studies of human face perception. I: Potentials generated in occipitotemporal cortex by face and non-face stimuli. Cereb Cortex 9:415-430.

Bellgowan PS, Buffalo EA, Bodurka J, Martin A (2009) Lateralized spatial and object memory encoding in entorhinal and perirhinal cortices. Learn Mem 16:433-438.

Boehm SG, Klostermann EC, Sommer W, Paller KA (2006) Dissociating perceptual and representation-based contributions to priming of face recognition. Conscious Cogn 15:163-174.

Brown MW, Aggleton JP (2001) Recognition memory: what are the roles of the perirhinal cortex and hippocampus? Nat Rev Neurosci 2:51-61.

Buffalo EA, Bellgowan PS, Martin A (2006) Distinct roles for medial temporal lobe structures in memory for objects and their locations. Learn Mem 13:638-643. 
DeSchepper B, Treisman A (1996) Visual memory for novel shapes: implicit coding without attention. J Exp Psychol Learn Mem Cogn 22:27-47.

Desimone R (1996) Neural mechanisms for visual memory and their role in attention. Proc Natl Acad Sci U S A 93:13494-13499.

Fiebach CJ, Gruber T, Supp GG (2005) Neuronal mechanisms of repetition priming in occipitotemporal cortex: spatiotemporal evidence from functional magnetic resonance imaging and electroencephalography. J Neurosci 25:3414-3422.

Friedman D, Johnson R Jr (2000) Event-related potential (ERP) studies of memory encoding and retrieval: a selective review. Microsc Res Tech 51:6-28.

Gabrieli JD (1998) Cognitive neuroscience of human memory. Annu Rev Psychol 49:87-115.

Grill-Spector K, Henson R, Martin A (2006) Repetition and the brain: neural models of stimulus-specific effects. Trends Cogn Sci 10:14-23.

Henson RN (2003) Neuroimaging studies of priming. Prog Neurobiol 70:53-81.

Henson R, Shallice T, Dolan R (2000) Neuroimaging evidence for dissociable forms of repetition priming. Science 287:1269-1272.

Horner AJ, Henson RN (2008) Priming, response learning and repetition suppression. Neuropsychologia 46:1979-1991.

James TW, Gauthier I (2006) Repetition-induced changes in BOLD response reflect accumulation of neural activity. Hum Brain Mapp $27: 37-46$

Kounios J, Smith RW, Yang W, Bachman P, D’Esposito M (2001) Cognitive association formation in human memory revealed by spatiotemporal brain imaging. Neuron 29:297-306.

Lamme VA, Roelfsema PR (2000) The distinct modes of vision offered by feedforward and recurrent processing. Trends Neurosci 23:571-579.

Musen G, Treisman A (1990) Implicit and explicit memory for visual patterns. J Exp Psychol Learn Mem Cogn 16:127-137.

Paller KA (1990) Recall and stem-completion priming have different electrophysiological correlates and are modified differentially by directed forgetting. J Exp Psychol Learn Mem Cogn 16:1021-1032.

Paller KA, Kutas M (1992) Brain potentials during memory retrieval provide neurophysiological support for the distinction between conscious recollection and priming. J Cogn Neurosci 4:375-391.

Paller KA, Wagner AD (2002) Observing the transformation of experience into memory. Trends Cogn Sci 6:93-102.

Paller KA, Kutas M, Mayes AR (1987a) Neural correlates of encoding in an incidental learning paradigm. Electroencephalogr Clin Neurophysiol 67:360-371.

Paller KA, Kutas M, Shimamura AP, Squire LR (1987b) Brain responses to concrete and abstract words reflect processes that correlate with later performance on a test of stem-completion priming. Electroencephalogr Clin Neurophysiol Suppl 40:360-365.

Paller KA, Hutson CA, Miller BB, Boehm SG (2003) Neural manifestations of memory with and without awareness. Neuron 38:507-516.

Peterson MA, Enns JT (2005) The edge complex: Implicit memory for figure assignment in shape perception. Percept Psychophys 67:727-740.

Richardson-Klavehn A, Bjork RA (1988) Measures of memory. Annu Rev Psychol 39:475-543.
Roediger HL 3rd (1990) Implicit memory. Retention without remembering. Am Psychol 45:1043-1056.

Rolls ET, Kesner RP (2006) A computational theory of hippocampal function, and empirical tests of the theory. Prog Neurobiol 79:1-48.

Rugg MD, Curran T (2007) Event-related potentials and recognition memory. Trends Cogn Sci 11:251-257.

Schacter DL, Buckner RL (1998) Priming and the brain. Neuron 20:185-195.

Schacter DL, Cooper LA, Delaney SM (1990) Implicit memory for unfamiliar objects depends on access to structural descriptions. J Exp Psychol Gen 119:5-24.

Schacter DL, Reiman E, Uecker A, Polster MR, Yun LS, Cooper LA (1995) Brain regions associated with retrieval of structurally coherent visual information. Nature 376:587-590.

Schacter DL, Wig GS, Stevens WD (2007) Reductions in cortical activity during priming. Curr Opin Neurobiol 17:171-176.

Schendan HE, Lucia LC (2010) Object-sensitive activity reflects earlier perceptual and later cognitive processing of visual objects between 95 and 500 ms. Brain Res 1329:124-141.

Schott BH, Richardson-Klavehn A, Henson RN, Becker C, Heinze HJ, Düzel E (2006) Neuroanatomical dissociation of encoding processes related to priming and explicit memory. J Neurosci 26:792-800.

Schott B, Richardson-Klavehn A, Heinze HJ, Düzel E (2002) Perceptual priming versus explicit memory: Dissociable neural correlates at encoding. J Cogn Neurosci 14:578-592.

Slotnick SD, Schacter DL (2006) The nature of memory related activity in early visual areas. Neuropsychologia 44:2874-2886.

Soldan A, Gazes Y, Hilton HJ, Stern Y (2008) Aging does not affect brain patterns of repetition effects associated with perceptual priming of novel objects. J Cogn Neurosci 20:1762-1776.

Squire LR (2004) Memory systems of the brain: a brief history and current perspective. Neurobiol Learn Mem 82:171-177.

Ungerleider LG, Haxby JV (1994) 'What' and 'where' in the human brain. Curr Opin Neurobiol 4:157-165.

van Turennout M, Ellmore T, Martin A (2000) Long-lasting cortical plasticity in the object naming system. Nat Neurosci 3:1329-1334.

Voss JL, Paller KA (2008a) Brain substrates of implicit and explicit memory: the importance of concurrently acquired neural signals of both memory types. Neuropsychologia 46:3021-3029.

Voss JL, Paller KA (2008b) Neural substrates of remembering: electroencephalographic studies. In: Learning and memory: a comprehensive reference (Byrne JH, ed), pp 79-97. Oxford: Elsevier.

Voss JL, Paller KA (2009a) An electrophysiological signature of unconscious recognition memory. Nat Neurosci 12:349-355.

Voss JL, Paller KA (2009b) Remembering and knowing: electrophysiological distinctions at encoding but not retrieval. Neuroimage 46:280-289.

Voss JL, Baym CL, Paller KA (2008) Accurate forced-choice recognition without awareness of memory retrieval. Learn Mem 15:454-459.

Wiggs CL, Martin A (1998) Properties and mechanisms of perceptual priming. Curr Opin Neurobiol 8:227-233. 